\title{
Less is (Just as Good as) More - an Investigation of Odor Intensity and Hedonic Valence in Mulsemedia QoE using Heart Rate and Eye Tracking
}

\author{
Gebremariam Mesfin, Estêvão B. Saleme, Oluwakemi Ademoye, Elahe Kani-Zabihi, Celso A.S. \\ Santos, and Gheorghiţă Ghinea, Member, IEEE
}

\begin{abstract}
Using olfactory media to enhance traditional multimedia content opens up novel opportunities for user interactions. Whilst the influence of olfaction on user experience in mulsemedia (multiple sensorial media) environments has been previously studied, the impact of the fundamental dimensions of scent intensity and valence (odor hedonic dimension or pleasantness) have been largely unexplored. This is precisely what we target in this paper, which reports the results of an empirical investigation examining how scent intensity and valence impact mulsemedia Quality of Experience (QoE). Accordingly, 54 participants were exposed to different odor valences and scent intensity levels when viewing three short multimedia clips. In particular, we examine both subjective (self-reported) as well as objective QoE metrics, as evidenced by user heart rates and eye gaze patterns. Results show that whilst eye gaze patterns are largely unaffected by the experimental conditions, valence does have a statistically significant impact upon user heart rates, as does intensity for two of the three clips employed in our study. In terms of subjective QoE, results indicate that hedonic valence impacts on the sense of reality and enjoyment; however varying odor intensity levels do not seem to differentially impact on user experience, bringing into question the need for strong scent intensities.
\end{abstract}

Index Terms - QoE, olfaction, hedonic valence, intensity, odor hedonic quality, mulsemedia, eye tracking, heart rate

\section{INTRODUCTION}

$\mathrm{I}_{\mathrm{a}}^{\mathrm{N}}$ $\mathrm{N}$ mulsemedia (multiple sensorial media), conventional audio-visual (AV) content is enhanced by incorporating additional components such as olfactory, gustatory and haptic stimuli (associated with the senses of smell, taste and touch respectively), thereby bringing new opportunities for the development of immersive technologies [22][41]. Research indicates that there are various characteristics associated with each such non-traditional media components such as crossmodality [12][31], intensity, and the ability to linger/waft [1], to name but a few.

In the context of media such as audio and images, their intensity (e.g. image brightness, loudness of audio) is important

This study was funded by the European Union's Horizon 2020 Research and Innovation program under Grant Agreement no. 688503. It was also was financed in part by the Coordenação de Aperfeiçoamento de Pessoal de Nível Superior - Brasil (CAPES) - Finance Code 88881.187844/2018-01. E. B. Saleme also acknowledges aid from the Federal Institute of Espírito Santo.

G. Mesfin and G. Ghinea are with the Department of Computer Science, Brunel University, UB8 3PH, United Kingdom (e-mail: \{gebremariam.assres; george.ghinea\}@brunel.ac.uk). for user perception and the associated user QoE. QoE comes from the achievement of users' expectations with regard to utility, the level of enjoyment considering their personalities, and their current state [7]. When it comes to nontraditional media, such as olfactory, it is remarkable that this property also holds [20], in spite of the fact that this latter medium has fundamental differences to audio and images. For instance, the spatial continuity of images and the temporal continuity of audio is not present in the case of olfactory media, which have a lingering characteristic.

One of the most exciting applications of olfaction is within the context of mulsemedia where olfactory media are used in conjunction with their more traditional audio-video counterparts [21][34][35][36]. Here, the application of smell in interactive systems has been increasingly explored aiming at finding out how to use this sense to immerse users in more realistic and engaging experiences. Whilst there has been a proliferation of studies related to the use of the olfactory modality in digital systems, there still remain some unexplored issues [22], linked to the incompletely comprehended features of scent intensities perceived by users in mulsemedia applications.

The other fundamental characteristic of odors, apart from intensity, is that of hedonic quality [6]. This represents the degree of pleasantness of odors and in this paper we use the term odor hedonic valence to denote its perceived quality as manifested by olfactory hedonic judgments in humans. Odor hedonic valence is a key property behind emotional valence reaction [30] and, whilst its impact on biophysical and emotional markers has been explored [19][30], the same cannot be said when it comes to uses of olfaction in mulsemedia scenarios (i.e. where three or more senses are engaged).

The study reported in this paper goes somewhat towards addressing this gap, by exploring the QoE impact of both olfactory intensity and valence in mulsemedia. Moreover, we adopt a two-pronged perspective when evaluating QoE, exploring it both from a subjective (self-reported) and objective (biophysical) standpoint. Indeed, whilst the former is

E.B. Saleme and C.A. Santos are with the Department of Informatics, Federal Institute of Espírito Santo, Brazil. E.B. Saleme was on PhD secondment to the Department of Computer Science, Brunel University (e-mail: \{estevaobissoli; celsoalbertosaibelsantos\}@gmail.com).

O. Ademoye is with the Faculty of Architecture, Computing and Engineering, University of Wales Trinity Saint David, United Kingdom (email:kemi.ademoye@uwtsd.ac.uk).

E. Kani-Zabihi is with the School of Computing and Engineering, University of West London, United Kingdom (e-mail: Elahe.Kani-Zabihi@uwl.ac.uk). 
ascertained through Likert scale responses, the latter is captured through user heart rates [25] and eye gaze paths [37]. In so doing, we believe that we obtain a more complete picture of QoE, and, with it, the potential to gain deeper insights into the user mulsemedia experience.

Accordingly, the structure of this paper is as follows. Section II reviews work related to the study undertaken, whilst Section III details its methodology. Section IV presents, analyzes, and discusses the results obtained; lastly, Section V draws conclusions and highlights conduits for future work.

\section{RELATED WORK}

The concept of QoE goes further than the so-called QoS (Quality of Service), also encompassing different users' attributes such as expectations, psychological profile, culture, among others [7][43] and it is rather difficult to capture given its multi-faceted nature. Moreover, the enrichment of multimedia applications with perceptual entities beyond those of sight and hearing seeks to improve the user's QoE. This is precisely the focus of mulsemedia applications, and much research has devoted efforts in this direction. Accordingly, studies in [2][3][21][47][49] indicated that the olfactory modality enhances users' QoE of viewing audio-visual content. The studies in [2][3][21] focused on investigating the impact of enhancing traditional audio-visual content with olfaction, while [47][49] further enhance audio-visual content with haptics and airflow in addition to olfaction. Findings from these studies have shown that olfaction in mulsemedia applications leads to an increased sense of reality and relevance [21], enjoyment [47], and the use of odors does not negatively impact on information assimilation [2]. Furthermore, Yuan et. al [49] demonstrated that the use of these multiple sensorial media sequences can partly mask a reduction in movie quality. Such congruent olfactory stimuli can be added into the audio-visual content through various mechanisms including cross-modal correspondence as described in [12] . Ademoye and Ghinea [1] and Murray et al. [34] showed that sensory effects affect a user's perception and tolerance to issues like content synchronization, and, by extension, QoE. The authors sought to determine tolerable time windows for the lack of synchronization between audiovisual content and olfactory stimuli during the multimedia presentations. Egan et al. [19] assessed users' QoE immersed in interactive environments from objective metrics by capturing physiological data such as HR (Heart Rate) and EDA (Electrodermal Activity). Research has also shown correlations between HR and odor pleasantness [5] and the use of scents in mulsemedia is thus unsurprising. To this end, Yuan et al.[49] demonstrated that the overall level of users' satisfaction is increased by up to $70 \%$ when adding mulsemedia content. In addition, Yuan et al. [48] carried out subjective analysis using different video qualities, with and without mulsemedia, to understand QoE in this context. They also proposed an adaptive strategy to select the best combination between video segments and sensory data for different bandwidth thresholds and users' requirement in order to improve QoE.

Whilst the positive impact of odors on mulsemedia QoE has been noted, it is surprising that the role of valence, a key emotional response to the pleasantness of odors, has in this context remains relatively unexplored [18]. This, in spite of the fact that it is to be expected that the impact of olfaction on QoE also depends (among other factors) on this particular hedonic dimension of odor quality. One of the few research endeavors which examined the impact of odor valences on $\mathrm{QoE}$ is that of Kroupi et al. [29]. In this study, authors have analyzed electroencephalogram (EEG) data of users experiencing hedonically different odors, whilst earlier work [46] of the authors had focused on classifying EEG signals to distinguish whether users had experienced pleasant (or not) odor stimuli. Related work has also examined the impact of multisensory inputs (heat, olfactory, as well as audio and visual cues) [15] as well as of odors of different hedonic dimensions on the sense of presence in Virtual Reality (VR) environments [4][26]. Zhang et al. [51] also explored the use of odors to enhance emotions in a game-playing setting, whilst highlighting the potential of odors for an increased QoE in games-based learning [11]. Moreover, Murray et al. [33] explored the perception of timing depending on the scent type (pleasant/unpleasant) presented to the participants. Authors concluded that the participants enjoyed pleasant smells than unpleasant ones irrespective of presentation timing.

Eye-tracking is a relatively under-utilized investigative channel in examining QoE. This is surprising, given that eyetracking devices are able to discover where human eyes are pointed given a reference and to capture users' behavior based on where they are gazing. Eye trackers are especially useful to determine how long a person is staring at different regions (user fixations) of a screen, which is distinctly useful when user QoE is influenced by cognitive tasks being undertaken. Indeed, Gulliver and Ghinea [24] showed that the use of eye tracking data provides cues for valuable insights. It has been applied as an input interface to several applications, i.e. to control a pointer on a computer screen [9][27][28]. However, its main application has been related to tasks involving research to understand users satisfaction/preferences [8][16] and cognitive studies to comprehend how a person behaves before making decisions [8][38] . Of direct relevance to our work, the ability of odors to enhance aspects of visual attention has been evidenced by previous eye-tracking studies [18][40]. The use of eye-tracking devices for research purposes requires some steps though. Perhaps the most important is the calibration process where precision measures, such as accuracy within the targeted area, are verified so as to obtain reliable data from the users' eyes movement [17]. In turn, the eyes' activity will aid to understand cognitive processes of low and high level with the end aim of either modeling complex cognitive processes or finding out content attractiveness.

It is to be noted that, whilst the QoE impact of different odor hedonic valences has been explored primarily from the perspective of their impact on EEG signals, not the same can be said when it comes to intensity - the other important dimension of odors. This, in spite of the fact that studies examining intensity in the context of psychophysics (such as [10], which showed that the olfactory perception of mild intensity odor 
leads to a decrease in low frequency and to an increase of the high frequency EEG components) and neurosciences (such as [45], which explored neural representations of odor intensity and affective valence) are not uncommon. Indeed, it is striking and baffling at the same time that, whilst odor intensity has been studied in a non-digital context, its effect and use in a digital context are sorely lacking.

This is precisely the niche that the study described in the current paper focuses on. Accordingly, it reports on the joint QoE impact of odor valence and intensity in a mulsemedia context (i.e. when odors accompany audio-visual content). To this end, we are now in a position to describe the methodology of the study undertaken, which we do next.

\section{Methodology}

\section{A. Participants}

A total of 54 participants (41 male, 13 female) participated in the experiment. They aged from the following average ranges: $41 \% 16-25,26 \% 26-35,24 \% 36-45,7 \% 46-55$, and $2 \%$ 56-65. Participants self-reported as being computer literate and all spoke English to (at least) the level needed to be educated in that language. Although participants were not tested about their olfactory sensibility, none reported anosmia (inability to sense smells); thus none were excluded from taking part in the study.

\section{B. Materials}

\section{1) Devices}

The devices used in the experiment consisted of a laptop, a screen monitor, a scent emitter, eye-tracking device, heart rate monitor, and a head positioner, as depicted in Figure 1. The laptop was a quad-core Intel Core i7-6700 HQ running at 2.6GHz, 16 GB RAM, 260 GB SSD, GTX 960M 4 GB GPU.

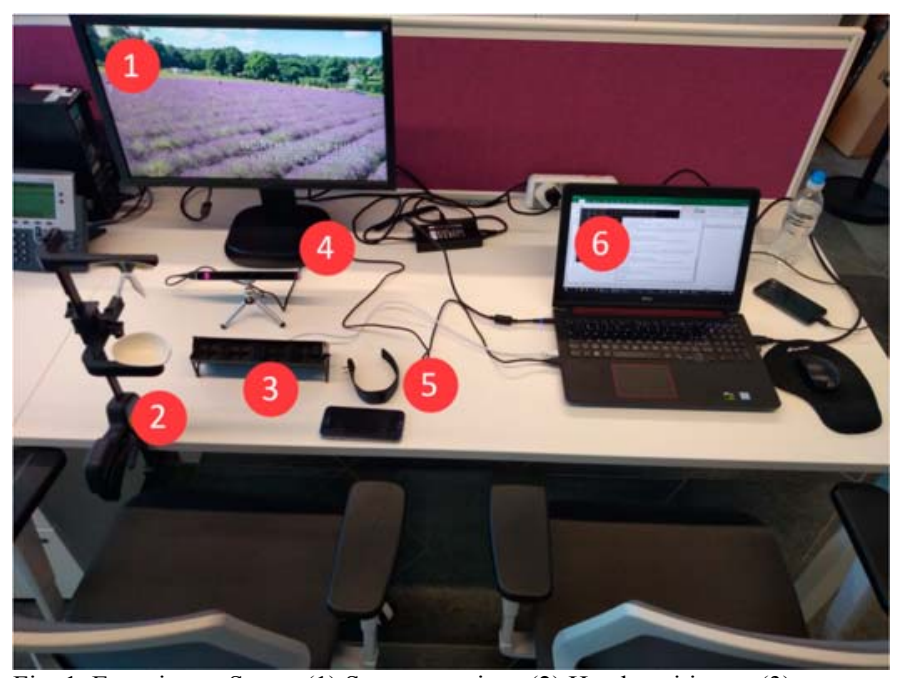

Fig. 1. Experiment Setup. (1) Screen monitor, (2) Head positioner, (3) Scent emitter, (4) Eye-tracking device, (5) Heart rate monitor, (6) Laptop

The scent emitter was from Exhalia - model SBi4. The eyetracking device was EyeTribe from The Eye Tribe. We chose to use the EyeTribe eye tracker because of previous reports that showed its accuracy in studies on gaze points and fixations
$[13][14][37]$. As for the heart rate monitor, the wristband Mio Link from Mio was employed. Commercially available\# and affordable wristband heart rate monitors have been shown to accurately measure heart rate and their use in research studies is on the rise [41][44][50]. Finally, the head positioner used was the ViewPoint QuickClamp from Arrington Research.

\section{2) Videos}

Each subject viewed three different one-minute long videos ${ }^{1}$. Lavender field comprised a walk-through fields of swaying lavender, Coffee ceremony depicted a coffee making video in Ethiopia, whilst Rollercoaster was a first person view of a roller coaster ride. All videos had the same 1920x1080 resolution and were played at 30 frames/second. Figure 2 contains snapshots of the three videos.
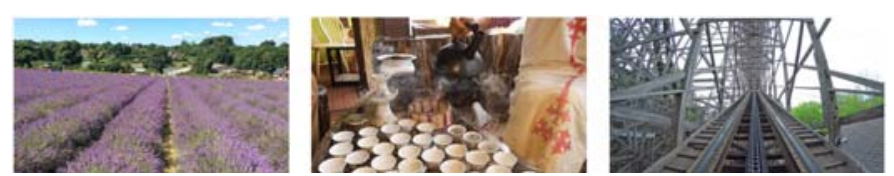

Fig. 2. Experimental Videos (from L-R: Lavender field, Coffee ceremony, Rollercoaster)

We limited the experiment to three videos to avoid exceeding the recommendation of 30 minutes per session in olfactory experiments as indicated by Murray et al. [32]. According to the authors, after 30 minutes, participants may experience olfactory adaptation, which could affect the consistency of findings.

\section{3) Scents}

Each of the three videos employed in our study was associated with a particular scent: Lavender field (lavender scent, positive hedonic valence), Coffee ceremony (coffee scent, medium hedonic valence), Rollercoaster (diesel scent, negative hedonic valence). Each experienced scent could have one of three different intensities: low, medium or high, cases in which the scent was respectively propagated by one, two or all fours fans of the Exhalia device. The choice of scents for each video was motivated by their content so that there was congruence between the scents and the videos; conversely the choice of videos was such so that their content was semantically congruent with the scents employed. Whilst for the lavender and coffee scents their use is self-evident, in the case of the diesel scent, justification lies in the mechanical association between this particular scent (reminiscent of lubricant aromas coupled with mildly pungent accents associated with burnt rubber) and the video content experienced. The fans, with corresponding scents, were switched on throughout the oneminute long playback of each video clip.

4) Software

The videos were annotated in MPEG-V, a standard to describe Sensory Effects Metadata (SEM); the laptop ran a video player called PlaySEM Sensory Effects Video Player that is based on VLC media player and compatible with MPEG-V standard. Furthermore, the laptop ran PlaySEM Sensory Effects Renderer 2 to process SEM and to control the scent emitter. Both software were introduced in [35]. 


\section{Experimental Design}

Two independent variables were manipulated: the odor intensity (with three different levels - low, medium, and high) and the odor hedonic valence (also with three levels - negative, medium, and positive). A mixed design was adopted whereby intensity was a between-subject variable and odor hedonic valence was a within-subjects variable. The choice of intensity as a between-subject variable is in line with other empirical studies exploring the impact of olfaction in mulsemedia [4][15][26] and is motivated by the desire for participants to be unaware of other potential intensities and thus, from this viewpoint, give blind/unbiased subjective judgements on their experiences. The dependent variable can be broadly termed as QoE, with two categories - objective (physiological) QoE, as manifested through heart rate and eye gaze patterns and subjective (self-reported) QoE as encompassed by responses to a QoE questionnaire. The motivation for using a two-pronged approach for measuring QoE is, as detailed in Section I, to get a more comprehensive and deeper view of mulsemedia QoE. Specifically, the choice of eye gaze and heart rate as objective QoE measures respectively stems from the ability of scents to enhance particular facets of visual attention [18][40] as well as the fact that previous research [5] has indicated that odor pleasantness does influence heart rate, and we wanted to explore if this also follows in a mulsemedia context. We now turn our attention to subjective QoE and in the next section we describe the questionnaire employed towards this end.

\section{QoE Questionnaire}

The QoE questionnaire comprised six questions targeting the user mulsemedia experience. Five of the six questions have been adopted from similar studies [1][2][21][33][34] investigating users' QoE of mulsemedia applications enhanced with olfactory effects. A sixth question was introduced to capture users' perception of the new dimension introduced in this study, the intensity of the olfactory effect. The response to each question was expressed on a 5-point Likert scale, as detailed below.

(1) Please rate the overall quality of the video clip.

\{Bad, Poor, Fair, Good, Excellent .

(2) How would you rate the intensity of the olfactory effects?

\{Too Weak, Weak, Just Fine, Strong, Too Strong\}.

(3) The olfactory effects enhance the sense of reality.

\{Strongly Agree, Agree, Neutral, Disagree, Strongly

Disagree .

(4) The olfactory effects are distracting.

\{Strongly Agree, Agree, Neutral, Disagree, Strongly

Disagree $\}$.

(5) The olfactory effects are annoying.

\{Strongly Agree, Agree, Neutral, Disagree, Strongly

Disagree .

(6) I enjoy watching the video with olfactory effects.

\{Strongly Agree, Agree, Neutral, Disagree, Strongly

Disagree .

\section{E. Procedure}

After being welcomed to the experimental room, participants were firstly briefed on the purpose of the experiment and asked whether they had any questions. Assuming that they were happy to go ahead with the experiments (and any questions they might have had had been satisfactorily answered), participants were asked to sit in front of the monitor by placing their chin on the chin rest of the head positioner, which ensured that the distance between them and the Exhalia device was at the recommended $0.5 \mathrm{~m} \mathrm{[32]} \mathrm{and} \mathrm{mainly} \mathrm{to} \mathrm{have} \mathrm{precise} \mathrm{data} \mathrm{from}$ the eye-tracking device. Moreover, for each participant, we had a calibration process, which consisted of verifying whether the level of calibration provided by the eye-tracking device was precise.

Each video was then played out to participants, with its corresponding scent. So as to counteract order effects, the presentation order of the clips was varied. Moreover, together with the presentation order, the associated intensities of the emitted scents were also varied, as detailed in Table I for the first nine participants (the allocation detailed in Table I was then cyclically repeated for the remainder of the user sample). Participants were, however, unaware at what particular intensity a scent was being emitted. After watching each clip, participants completed the QoE questionnaire detailed above after which each participant proceeded to watch the next video clip, but not before 60 s had elapsed. This was so that any lingering odors from the previously watched video would have dispersed, in line with empirical recommendations for conducting olfactory-enhanced multimedia experiments [32]. Finally, at the end of the experiment, qualitative opinions on the overall experience were also collected from participants.

TABLE I

Allocation of PaRTICIPANTS TO VIDEO (ODOR INTENSITY); L=LOW; $\mathrm{M}=$ MEDIUM; $\mathrm{H}=\mathrm{HIGH}$ INTENSITY

\begin{tabular}{|c|c|c|c|}
\hline Id & Video 1 & Video 2 & Video 3 \\
\hline 1 & Rollercoaster $\{\mathrm{L}\}$ & Lavender $\{\mathrm{M}\}$ & Coffee $\{\mathrm{H}\}$ \\
\hline 2 & Coffee $\{\mathrm{M}\}$ & Rollercoaster $\{\mathrm{H}\}$ & Lavender $\{\mathrm{L}\}$ \\
\hline 3 & Lavender $\{\mathrm{H}\}$ & Coffee $\{\mathrm{L}\}$ & Rollercoaster $\{\mathrm{M}\}$ \\
\hline 4 & Rollercoaster $\{\mathrm{M}\}$ & Lavender $\{\mathrm{H}\}$ & Coffee $\{\mathrm{L}\}$ \\
\hline 5 & Coffee $\{\mathrm{H}\}$ & Rollercoaster $\{\mathrm{L}\}$ & Lavender $\{\mathrm{M}\}$ \\
\hline 6 & Lavender $\{\mathrm{L}\}$ & Coffee $\{\mathrm{M}\}$ & Rollercoaster $\{\mathrm{H}\}$ \\
\hline 7 & Rollercoaster $\{\mathrm{H}\}$ & Lavender $\{\mathrm{L}\}$ & Coffee $\{\mathrm{M}\}$ \\
\hline 8 & Coffee $\{\mathrm{L}\}$ & Rollercoaster $\{\mathrm{M}\}$ & Lavender $\{\mathrm{H}\}$ \\
\hline 9 & Lavender $\{\mathrm{M}\}$ & Coffee $\{\mathrm{H}\}$ & Rollercoaster $\{\mathrm{L}\}$ \\
\hline
\end{tabular}

\section{RESULTS AND DISCUSSION}

All responses from the QoE questionnaire involved mapping the 5 Likert scale items to the integer values of 1 to 5 for analysis purposes. Data were analyzed with the Statistical Package for the Social Sciences (SPSS) for Windows version (release 25.0). Analysis of MOS (Mean Opinion Score) data with the Shapiro-Wilk test and of the heart rate data with the Kolmogorov-Smirnoff test for normality revealed p-values less than 0.05, highlighting that the data was not normally distributed. Consequently, we employed non-parametric tests to analyze the data [23]. Accordingly, significance of the difference in MOS and heart rate readings between odor hedonic valence and intensity was analyzed using the KruskalWallis and Friedman (non-parametric) tests, respectively. A significance level of $\mathrm{p}<0.05$ was adopted for the study.

Tables II and III present descriptive statistics of the responses 
on QoE questionnaire for hedonic valence and odor intensity. Tables V, VI, VII, and VIII, and graphs depicting heart rate variation with respect to both odor, intensity, and their interaction are also presented (Figures 4, 7, and 8).

TABLE II

DESCRIPTIVE STATISTICS FOR ODOR HEDONIC VALENCE

\begin{tabular}{|c|c|c|c|c|}
\hline \multirow[t]{2}{*}{ Dependent Variable } & \multicolumn{4}{|c|}{ Hedonic Valence } \\
\hline & Type & Mean & SD & SE \\
\hline \multirow[t]{3}{*}{ Video Quality } & Negative & 4.30 & 0.743 & 0.101 \\
\hline & Medium & 4.43 & 0.690 & 0.094 \\
\hline & Positive & 4.35 & 0.677 & 0.092 \\
\hline \multirow{3}{*}{ Perceived Intensity } & Negative & 3.31 & 0.948 & 0.129 \\
\hline & Medium & 3.41 & 0.74 & 0.101 \\
\hline & Positive & 3.44 & 0.839 & 0.114 \\
\hline \multirow[t]{3}{*}{ Enhanced Reality } & Negative & 3.78 & 1.076 & 0.146 \\
\hline & Medium & 4.20 & 0.877 & 0.119 \\
\hline & Positive & 4.28 & 0.856 & 0.116 \\
\hline \multirow[t]{3}{*}{ Distraction } & Negative & 3.70 & 1.075 & 0.146 \\
\hline & Medium & 3.91 & 0.830 & 0.113 \\
\hline & Positive & 3.91 & 0.976 & 0.133 \\
\hline \multirow[t]{3}{*}{ Annoyance } & Negative & 3.81 & 1.117 & 0.152 \\
\hline & Medium & 4.15 & 0.960 & 0.131 \\
\hline & Positive & 4.19 & 0.870 & 0.118 \\
\hline \multirow[t]{3}{*}{ Enjoyment } & Negative & 3.67 & 1.229 & 0.167 \\
\hline & Medium & 4.17 & 1.005 & 0.137 \\
\hline & Positive & 4.31 & 0.907 & 0.123 \\
\hline
\end{tabular}

TABLE III

DESCRIPTIVE STATISTICS FOR ODOR INTENSITY

\begin{tabular}{lllll}
\multirow{2}{*}{ Dependent Variable } & \multicolumn{4}{c}{ Odor Intensity } \\
\cline { 2 - 5 } Video Quality & Level & Mean & SD & SE \\
\hline \multirow{5}{*}{ Perceived Intensity } & Low & 4.20 & 0.762 & 0.104 \\
& Hedium & 4.43 & 0.602 & 0.082 \\
\cline { 2 - 5 } Enhanced Reality & Low & 4.44 & 0.718 & 0.098 \\
\hline \multirow{5}{*}{ Distraction } & Medium & 3.44 & 1.002 & 0.136 \\
& High & 3.43 & 0.716 & 0.108 \\
\cline { 2 - 5 } & Low & 3.94 & 1.017 & 0.138 \\
& Medium & 4.07 & 1.061 & 0.144 \\
& High & 4.24 & 0.775 & 0.106 \\
\cline { 2 - 5 } Annoyance & Low & 3.87 & 0.912 & 0.124 \\
& Medium & 3.76 & 1.027 & 0.140 \\
& High & 3.89 & 0.965 & 0.131 \\
\cline { 2 - 5 } Enjoyment & Low & 4.19 & 0.892 & 0.121 \\
& Medium & 3.96 & 1.132 & 0.154 \\
& High & 4.00 & 0.952 & 0.130 \\
\hline & Low & 3.93 & 1.113 & 0.152 \\
& Medium & 4.09 & 1.202 & 0.164 \\
& High & 4.13 & 0.933 & 0.127 \\
\hline \multirow{2}{*}{ Eow } & & & &
\end{tabular}

\section{A. The Impact of Odor Hedonic Valence}

The expectation for odor hedonic valence was that the level of self-reported opinion about positive dependent variables would increase and negative feelings would decrease even for non-positive valences. Table II presents the Mean, SD (Standard Deviation) and SE (Standard Error) values for the dependent variables from the perspective of hedonic valence. These values suggest that the more positive the odor hedonic dimension, the more sensitive the participants were on average.

Additional analysis running the Friedman test indicated that there was a statistically significant difference for Enhanced Reality $\left(\chi^{2}(2)=7.390, \mathrm{p}=0.025\right)$, Annoyance $\left(\chi^{2}(2)=6.136\right.$, $\mathrm{p}=0.047)$, and Enjoyment $\left(\chi^{2}(2)=11.774, \mathrm{p}=0.003\right)$ depending on which type of valence was experienced.

However, there were no statistically significant differences for Video Quality $\left(\chi^{2}(2)=0.787, \mathrm{p}=0.675\right)$, Perceived Intensity $\left(\chi^{2}(2)=1.163, \quad \mathrm{p}=0.559\right), \quad$ and Distraction $\quad\left(\chi^{2}(2)=1.068\right.$, $\mathrm{p}=0.586)$.

After asking about the content of the videos and the whole experience with olfactory effects, we tried to capture personal feelings of participants. The perceived congruence of the smell and the content in the Roller coaster video (negative hedonic valence) was particularly peculiar. Some participants reported they were expecting fresh air and smell of wood, mainly the ones who had been on rollercoasters before, whereas others enjoyed the smell. In the Coffee ceremony video (medium hedonic valence), some participants expressed they do not like coffee, however, they enjoyed the video with that smell as if it was coming into their direction during the coffee ceremony. Others mentioned a feeling of hungry after watching it. With regard to the Lavender field video (positive hedonic valence), there was a common sense that it was pleasant.

Analysis of heart rate readings was also performed with respect to the hedonic dimension of odors. The mean heart rate readings of participants recorded while watching the 60-second sample video clips is depicted in the line plot of Figure 3 . The captured data were grouped by odors hedonic values (negative, medium and positive). The figure indicates that, generally, the heart rate readings for the negative and medium odor hedonic values are high and low respectively, the positive value being in between. In addition, a Friedman test was performed to determine if heart rate reading was affected for three groups of users who watched videos with three different odor hedonic values: negative $(n=3230)$; medium $(n=3227)$; and positive $(n=3197)$. Table IV shows the Friedman mean ranks for the three video clips.

At a significance level of $\mathrm{p}<0.05$, the test showed that there was a statistically significant difference in heart rate readings between the three groups $\left(\left(\chi^{2}(2)=98.632, \mathrm{p}=0.000\right)\right.$. This implies that odor hedonic valence, or the degree of pleasantness of odor stimuli, can have a significant influence on QoE as perceived in the difference in heart rate readings.

TABLE IV

FRIEDMAN TEST MEAN RANKS FOR EACH OdOR.

\begin{tabular}{llc}
\hline Odor Hedonic Valence & N & Mean Rank \\
\hline Positive (Lavender field) & 3197 & 1.95 \\
Medium (Coffee ceremony) & 3227 & 1.91 \\
Negative (Rollercoaster) & 3230 & 2.14 \\
\hline
\end{tabular}

\section{B. Does Olfactory Intensity Count?}

As for intensity, it was expected that the stronger the intensity, the more positive would be the impact on the dependent variables, even though it could supposedly increase 
distraction and eventually cause some irritation. However, the first results depicted in Table III did not confirm it. By examining the values shown on this table, there is no relevant pattern detected after increasing the intensity of the smell presented to the participants.

Further analysis running the Kruskal-Wallis test showed that there were no statistically significant difference between median scores for groups of intensity for Video Quality $\left(\chi^{2}(2)=3.751, \mathrm{p}=0.153\right)$, Perceived Intensity $\left(\chi^{2}(2)=0.911\right.$, $\mathrm{p}=0.634)$ Enhanced Reality $\left(\chi^{2}(2)=2.011, \quad \mathrm{p}=0.366\right)$, Distraction $\left(\chi^{2}(2)=0.407, \mathrm{p}=0.816\right)$, Annoyance $\left(\chi^{2}(2)=1.224\right.$, $\mathrm{p}=0.542)$, and Enjoyment $\left(\chi^{2}(2)=1.549, \mathrm{p}=0.461\right)$.

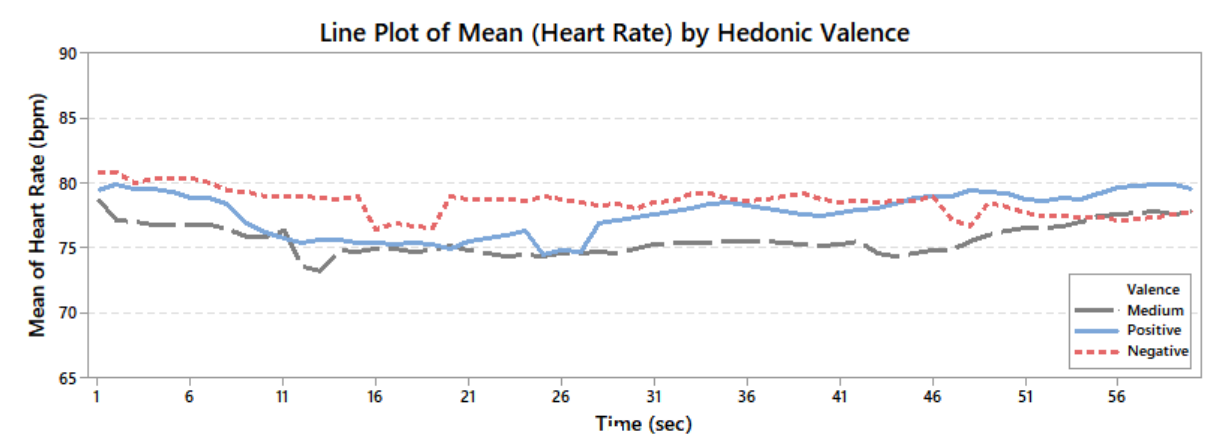

Interval Plot of Heart Rate by Valence $95 \%$ Bonferroni $\mathrm{Cl}$ for the Mean

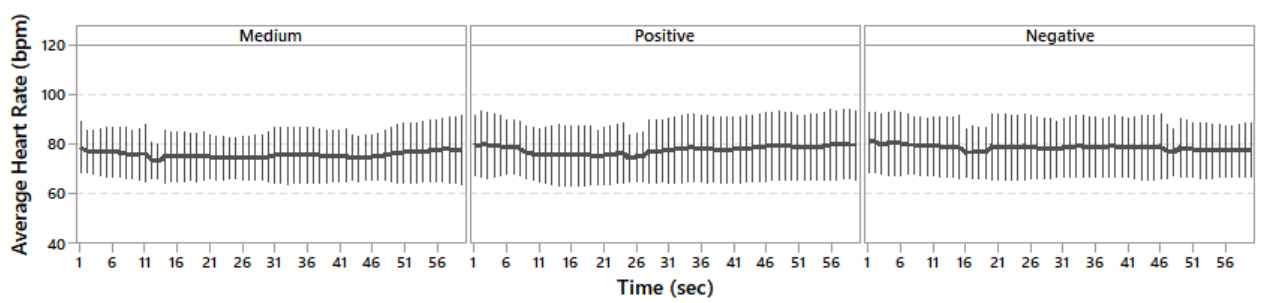

Fig. 3. Mean heart rate readings with respect to the hedonic valence of odors

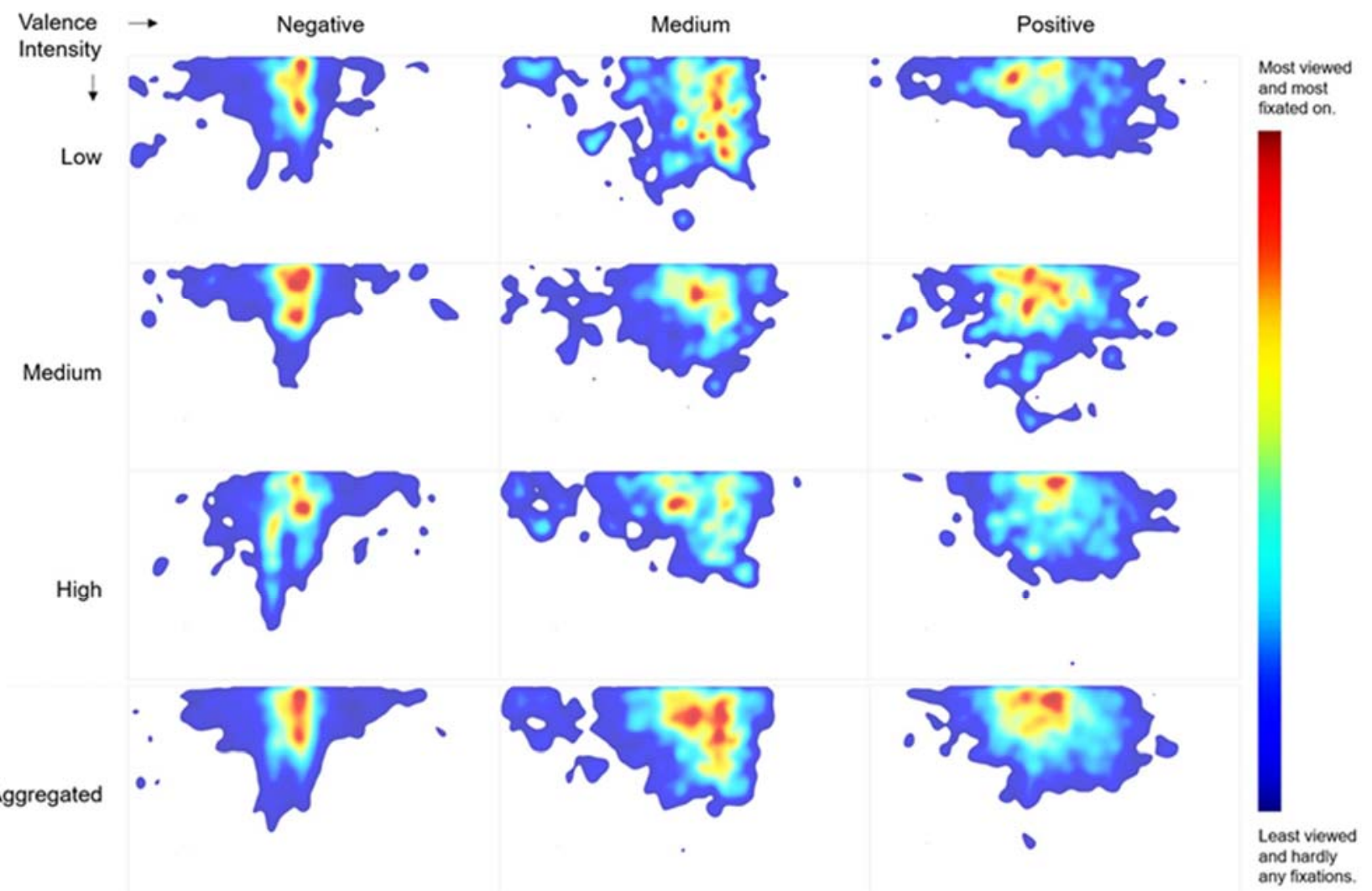

Fig. 4. General heat map across the hedonic valences for each odor intensity and aggregation by hedonic valence. 
TABLE V

KRUSKAL-WALLIS TEST MEAN RANKS FOR INTENSITY (ROLLERCOASTER).

\begin{tabular}{llc}
\hline Intensity & $\mathrm{N}$ & Mean Rank \\
\hline Low & 1078 & 1644.77 \\
Medium & 1072 & 1452.88 \\
High & 1072 & 1743.57 \\
\hline
\end{tabular}

TABLE VI

KRUSKAL-WALLIS TEST MEAN RANKS FOR INTENSITY (COFFEE CEREMONY).

\begin{tabular}{llc}
\hline Intensity & $\mathrm{N}$ & Mean Rank \\
\hline Low & 1077 & 1543.97 \\
Medium & 1054 & 1832.74 \\
High & 1066 & 1423.49 \\
\hline
\end{tabular}

TABLE VII

KRUSKAL-WALLIS TEST MEAN RANKS FOR INTENSITY (LAVENDER FIELD).

\begin{tabular}{llc}
\hline Intensity & $\mathrm{N}$ & Mean Rank \\
\hline Low & 1074 & 1656.28 \\
Medium & 1080 & 1567.66 \\
High & 1076 & 1622.82 \\
\hline
\end{tabular}

TABLE VIII

KRUSKAL-WALLIS TEST MEAN RANKS FOR INTENSITY (AGGREGATE).

\begin{tabular}{lcl}
\hline Intensity & $\mathrm{N}$ & Mean Rank \\
\hline Low & 3229 & 4833.71 \\
Medium & 3206 & 4864.89 \\
High & 3219 & 4784.04 \\
\hline
\end{tabular}

TABLE IX

KRUSKAL-WALLIS TEST STATISTICS FOR INTENSITY FOR EACH VIDEO CLIPS AND ON AGGREGATE

\begin{tabular}{llll}
\hline Video & $\chi^{2}$ & $\mathrm{df}$ & $\mathrm{p}$ \\
\hline Rollercoaster & 54.113 & 2 & 0.000 \\
Coffee Ceremony & 110.053 & 2 & 0.000 \\
Lavender & 4.966 & 2 & 0.083 \\
Aggregate & $\mathbf{1 . 3 7 7}$ & $\mathbf{2}$ & $\mathbf{0 . 5 0 2}$ \\
\hline
\end{tabular}

Complementarily, Figure 4 presents heat maps for each hedonic valence for the three levels of odor intensity. This conveys the message to where participants gazed at whilst watching the videos. Heat maps can be useful to show patterns for different groups. We used EyeTribe UI and EyeTribe Server ${ }^{2}$, both provided by the creator of the eye-tracking device, to record eye gaze data following the procedure described in Section III-E. The data was captured exactly when the video started and finished for each user. EyeTribe Server generated a JSON (JavaScript Object Notation) file for each session, which was then processed by a Java routine to obtain the $(x, y)$ coordinates separated by user, video, and scent intensity. Finally, the heat maps were plotted using a Python package for handling eye-tracking data created by Dalmaijer et al. [14]. In agreement with previous tests run for intensity, they showed that, when it varies, participants' gaze-behavior changes slightly but not enough to create new significant different patterns. For negative hedonic valence, the heat maps suggest that the focal point was the track and it did not vary significantly. For medium hedonic valence, their eyes were focused mostly on the movements of the household woman handling a jar and pouring the coffee to the cups. For positive hedonic valence, the participants focused on the top central area, just above the lavender field, with some slight variations.

What is to be especially remarked is that, as Table III highlights, Perceived Intensity values, no matter what the actual intensity employed was, hovered around the mid, "Just Fine", value. These findings were further confirmed by qualitative feedback which participants gave, some of whom declared a sense of a weak smell at the presence of medium and high intensities, whilst others pointed out they felt strong smell intensity even when a weak one had actually been employed. Our results would thus seem to suggest that, in contrast to hedonic valence, the actual emitted intensity matters not so much on user enjoyment of mulsemedia, as long as one, of course, incorporates olfactory effects in such presentations.

Analysis of heart rate readings was also performed with respect to odor intensity. The mean heart rate readings of participants recorded while watching the three 60 -second video clips is depicted in the charts in Figure 5. The readings in each of the videos are grouped by the level of odor intensity (low, medium and high). The charts show that the impact of odor intensity on heart rate readings differs in each of the video clips. For example, highest hear-rate readings can be observed for medium, high, and low odor intensity levels in the Lavender field, Coffee ceremony, and Rollercoaster videos, respectively. In addition, a Kruskal-Wallis test was conducted to determine if heart rate readings were different for each of the three groups of users who watched the video clips with three levels of odor intensities. Tables IV-VIII show the Kruskal-Wallis mean ranks for each of the three video clips and an aggregate for each odor intensity levels (low, medium, and high), respectively.

As can be seen in Table IX, a statistically significant difference is observed between the intensity levels for each of the Rollercoaster $\left(\chi^{2}(2)=54.113, \mathrm{p}=0.000\right)$, and Coffee ceremony $\left(\chi^{2}(2)=110.053, p=0.000\right)$ video clips; however, the difference for Lavender field is not significant $\left(\chi^{2}(2)=4.966\right.$, $\mathrm{p}=0.083$ ). This implies that, at the $\mathrm{p}<0.05$, odor intensity has significant influence on QoE in each of the video clips (except Lavender field) as perceived in the difference in heart rate readings. However, on the table, the aggregate statistical test result shows insignificant difference in heart rate depending on which level of odor intensity was used $\left(\chi^{2}(2)=1.377, p=0.502\right)$ which implies (generally) the influence of odor intensity on $\mathrm{QoE}$ is insignificant as perceived in the difference in heart rate readings.

\section{Interaction between Odor Hedonic Valence and Intensity}

A two-way ANOVA was conducted that examined the effect of valence and intensity level on QoE. There was no statistically significant interaction between the effects of valence and intensity level on Video Quality $(\mathrm{F}(4,153)=0.707, \mathrm{p}=0.588)$, 
Perceived Intensity $(\mathrm{F}(4,153)=0.623, \mathrm{p}=0.647)$, Enhanced Reality $(\mathrm{F}(4,153)=0.400, \mathrm{p}=0.809)$, Distraction $(\mathrm{F}(4,153)=$ $0.413, \mathrm{p}=0.799)$, Annoyance $\mathrm{F}(4,153)=0.884, \mathrm{p}=0.475)$, Enjoyment $(\mathrm{F}(4,153)=1.193, \mathrm{p}=0.316)$.

We observed that, on average, the participants reported that Video Quality is perceived slightly better in negative and medium hedonic valences with medium and high odor intensities. The Perceived Quality for all the hedonic valences is affected with medium and high intensities of smell, albeit there is no pattern. Enhanced Reality increases according to the hedonic valence, but again, there is no interaction between the former and odor intensity. As for Distraction and Annoyance, though there is apparently a reduction of their scores under higher odor intensities for negative hedonic valences, once more there is no relevant pattern observed. Finally, the levels of Enjoyment are higher when the hedonic valence is favorable. However, the connection between hedonic valence and odor intensity is not evident based on the experimental data.
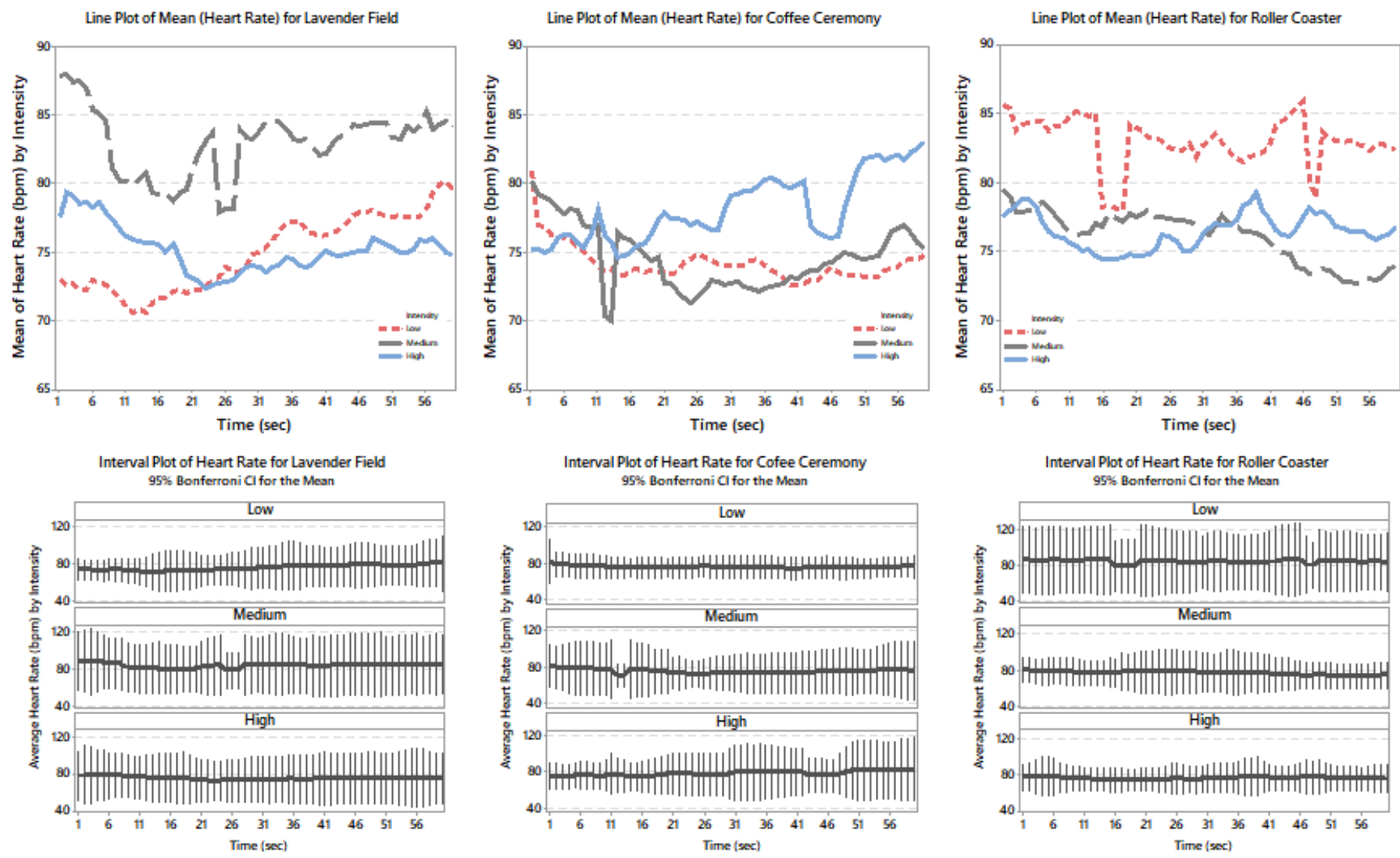

Fig. 5. Mean heart rate readings with respect to intensity of odors for each of the video clips.

\section{Notes on Multisensory Systems' Design}

The findings of the impact of odor hedonic valence and intensity in olfaction-based mulsemedia provide the following design recommendations:

- Content attractiveness/averseness should be carefully assessed. The content presented through the system and the user's expectation prevail over the level of intensity of the delivered scent.

- Devices' scent diffusing capacity might be important but the trade-off between QoE and development cost needs to be considered. Olfactory experiences will enrich QoE but the diffusing capacity provided by the olfactory device in terms of intensity will not be a determinant factor.

- Scent intensity does not need to be over-thought whilst authoring sensory effects. Sensory effects authoring requires time and might be costly, therefore, as scent intensity is not likely to severely affect QoE, this stage can be done without overelaboration on odor intensity.

\section{CONCLUSION}

This work investigated the impact of odor hedonic valence and intensity levels on QoE for olfaction-based mulsemedia systems and proposed some guidelines for their design based on the study findings. To the best of our knowledge, this is the first time that the impact of scent intensity on QoE has been reported in the literature. As for odor hedonic valence, it was found that it plays an important role in the sense of reality and enjoyment when exposed to olfactory experiences. The more positive, the better the mulsemedia QoE. Moreover, there was a statistically significant difference for the self-reported annoyance. As far as intensity is concerned, we showed that QoE is not statistically significant impacted by its values in our configuration; we, therefore, propose the use of low-intensity odor stimuli in 
mulsemedia setups. Not only does this economize resources, the user QoE will not be significantly affected.

Furthermore, an analysis of the interaction between odor hedonic valence and intensity did not evidence a bond between them in olfaction-based mulsemedia. Therefore, it leads us to conclude that expectation over the experience that is about to start influences QoE more than merely adjusting the intensity of the scent. Unlike the volume of a sound for hearing and the color attributes for sight, the extent of odors delivered to the user's nose is far more subjective. Thereby, there is no need to have overwhelming scents in olfaction-based mulsemedia.

Future work can be directed to address the lack of orthogonal/independent dimensions of smell and how best to leverage this in a mulsemedia context, which is one of its key challenges. Whilst our study has shed an interesting perspective in respect of integrating olfaction in mulsemedia applications, we also recognize that the future use of devices with a higher specification to measure physiological signals could reveal further novel insights. We also recognize that valence can be assessed not only by measuring physiological parameters, as done in our research, but also through self-reported measures such as pick-a-mood, self-assessment manikin (SAM), and these can be part of future endeavors. Last but not least, it is worth mentioning that the main finding of our exploratory study, namely that odor intensity does not significantly impact mulsemedia QoE, needs to be further validated, and one of the ways this could be done is within the confines of an empirical study incorporating a within-subjects experimental design.

\section{REFERENCES}

[1] O. A. Ademoye and G. Ghinea. 2009. Synchronization of olfactionenhanced multimedia. IEEE Transactions on Multimedia, 11, 3, $561-565$.

[2] O. A. Ademoye and G. Ghinea. 2013. Information recall task impact in olfaction-enhanced multimedia. ACM Transactions on Multimedia Computing, Communications, and Applications (TOMM), 9, 317.

[3] O. A. Ademoye, N. Murray, G-M. Muntean, and G. Ghinea. 2016. Audio masking effect on inter-component skews in olfactionenhanced multimedia presentations. ACM Trans. on Multimedia Computing, Communications, and Applications (TOMM),12, 451.

[4] O. Baus and S. Bouchard. 2017. Exposure to an Unpleasant Odour Increases the Sense of Presence in Virtual Reality. Virtual Reality 21, 2, 59-74.

[5] M. Bensafi, C. Rouby, V. Farget, B. Bertrand, M. Vigouroux, and A. Holley. 2002. Autonomic nervous system responses to odors: the role of pleasantness and arousal. Chemical Senses, 27(8), 703-709.

[6] C. M. Brendl and E. T. Higgins. 1996. Principles of judging valence: What makes events positive or negative? In Advances in experimental social psychology. Vol. 28. Elsevier, 95-160.

[7] K. Brunnström, S. A. Beker, K. De Moor, A. Dooms, S. Egger, MN. Garcia, T. Hossfeld, S. Jumisko-Pyykkö, C. Keimel, M-C. Larabi, B. Lawlor, P. Le Callet, S. Möller, F. Pereira, M. Pereira, A. Perkis, J. Pibernik, A. Pinheiro, A. Raake, P. Reichl, U Reiter, R. Schatz, P. Schelkens, L. Skorin-Kapov, D. Strohmeier, C. Timmerer, M. Varela, I. Wechsung, J. You, and A. Zgank.2013. Qualinet White Paper on Definitions of Quality of Experience. https://hal.archives-ouvertes.fr/hal-00977812Fifth Qualinet meeting, Novi Sad, March 12, 2013.

[8] G. Buscher, R. Biedert, D. Heinesch, and A. Dengel. 2010. Eye tracking analysis of preferred reading regions on the screen. In CHI'10 Extended Abstracts on Human Factors in Computing Systems . ACM, 3307-3312.

[9] M. C. Chen, J. R. Anderson, and M. H. Sohn. 2001. What can a mouse cursor tell us more?: correlation of eye/mouse movements on web browsing. In CHI'01 extended abstracts on Human factors in computing systems . ACM, 281-282.

[10] A. A. Cherninskii, I. G. Zima, N. Y. Makarchouk, N. G. Piskorskaya, and S. A. Kryzhanovskii. 2009. Modifications of EEG related to directed perception and analysis of olfactory information in humans. Neurophysiology 41, 1, 63-70.

[11] A. Covaci, G. Ghinea, C-H. Lin, S-H. Huang, and J-L. Shih. 2018. Multisensory games-based learning - lessons learnt from olfactory enhancement of a digital board game. Multimedia Tools and Applications 77, 16, 21245-21263.

[12] A. Covaci, G. Mesfin, N. Hussain, E. Kani-Zabihi, F. Andres, and G. Ghinea. 2018. A study on the quality of experience of crossmodal mulsemedia. In Proc. 10th International Conference on Management of Digital EcoSystems ACM, 176-182.

[13] E. S. Dalmaijer. Is the low-cost eyetribe eye tracker any good for research? PeerJ PrePrints, 2014.

[14] E. S. Dalmaijer, S. Mathôt, and S. Van der Stigchel. 2014. PyGaze: An open-source, cross-platform toolbox for minimal- effort programming of eyetracking experiments. Behavior research methods 46, 4, 913-921.

[15] H.Q. Dinh, N. Walker, L.F. Hodges, C. Song, and A. Kobayashi. 1999. Evaluating the Importance of Multi-Sensory Input on Memory and the Sense of Presence in Virtual Environments. IEEE Virtual Reality. pp. 222-228.

[16] S. Djamasbi, T. Tullis, J. Hsu, E. Mazuera, K. Osberg, and J. Bosch. 2007. Gender preferences in web design: usability testing through eye tracking. AMCIS 2007 Proceedings (2007), 133.

[17] A. T. Duchowski. 2007. Eye tracking methodology. Theory and practice 328.

[18] K. Durand, J.Y. Baudouin, D.J. Lewkowicz, N. Goubet, and B. Schaal, B. 2013. Eye-catching odors: olfaction elicits sustained gazing to faces and eyes in 4-month-old infants. PLoS One, 8(8), e70677

[19] D. Egan, S. Brennan, J. Barrett, Y. Qiao, C. Timmerer, and N. Murray. 2016. An evaluation of Heart Rate and ElectroDermal Activity as an objective QoE evaluation method for immersive virtual reality environments. In 2016 Eighth International Conference on Quality of Multimedia Experience (QoMEX) . 1-6.

[20] J. Frasnelli. [n. d.]. On the way to understand how we perceive odors. https://bit.ly/2Pi4FLo, accessed 2018-11-29.

[21] G. Ghinea and O. Ademoye. 2012. The sweet smell of success: Enhancing multimedia applications with olfaction. ACM Trans. on Multimedia Comput. Commun. Appl. (TOMM) 8, 1 (2012), 2.

[22] G. Ghinea, C. Timmerer, W. Lin, and S. R. Gulliver. 2014. Mulsemedia: State of the Art, Perspectives, and Challenges. ACM Trans. Multimedia Comput. Commun. Appl. (TOMM) 11, 1s, Article 17,23 pages.

[23] J. Greene and M. D’Oliveira. 2005. Learning to use statistical tests in psychology . McGraw-Hill Education (UK).

[24] S. R. Gulliver and G. Ghinea. 2004. Stars in their eyes: what eyetracking reveals about multimedia perceptual quality. IEEE Transactions on Systems, Man, and Cybernetics - Part A: Systems and Humans 34, 4, 472-482.

[25] N. Hussain, G. Mesfin, A. Covaci, and G. Ghinea. 2018. Towards Augmenting Multimedia QoE With Wearable Devices: Perspectives from an Empirical Study. In 2018 IEEE Int. Conf. on Multimedia \& Expo Workshops (ICMEW), IEEE, 1-6.

[26] L. Jones, C.A. Bowers, D. Washburn, A. Cortes, and R.V. Satya. 2004. Effect of Olfaction on Immersion into Virtual Environments. Human performance, situation awareness and automation: Issues and considerations for the $21^{\text {st }}$ century, pp. 282-285.

[27] E. Y. Kim, S. K. Kang, K. Jung, and H. J. Kim. 2005. Eye mouse: mouse implementation using eye tracking. In 2005 Digest of Technical Papers. International Conference on Consumer Electronics, 2005. ICCE. 207-208.

[28] T. Kocejko, A. Bujnowski, and J. Wtorek. 2009. Eye-Mouse for Disabled. Springer Berlin Heidelberg, Berlin, Heidelberg, 109-122.

[29] E. Kroupi, A. Yazdani, J-M. Vesin, and T. Ebrahimi. 2014. EEG correlates of pleasant and unpleasant odor perception. ACM Trans. on Multimedia Comput. Commun. Appl. (TOMM) 11, 1s (2014), 13.

[30] J. N. Lundström, S. Seven, M. J. Olsson, B. Schaal, and T. Hummel, 2006. Olfactory event-related potentials reflect individual differences in odor valence perception. Chemical senses, 31, 8, 705-711. 
[31] G. Mesfin, N Hussain, A. Covaci, and G. Ghinea. 2018. Inverse and Transitivity of Cross-Modal Correspondence in Mulsemedia. In 2018 IEEE International Conference on Multimedia \& Expo Workshops (ICMEW), IEEE, 1-6.

[32] N. Murray, O. A. Ademoye, G. Ghinea, and G-M. Muntean. 2017. A tutorial for olfaction-based multisensorial media application design and evaluation. ACM Computing Surveys (CSUR) 50, 5, 67.

[33] N. Murray, B. Lee, Y. Qiao, and G. Miro-Muntean. 2017. The Impact of Scent Type on Olfaction-Enhanced Multimedia Quality of Experience. IEEE Transactions on Systems, Man, and Cybernetics: Systems, 47, 9, 2503-2515.

[34] N. Murray, Y. Qiao, B. Lee, A. K. Karunakar, and G-M. Muntean. 2013. Subjective Evaluation of Olfactory and Visual Media Synchronization. In Proc. 4th ACM Multimedia Systems Conference (MMSys '13), 162-171.

[35] N. Murray, Y. Qiao, B. Lee, and G-M. Muntean. $2014 . \quad$ Userprofile-based perceived olfactory and visual media synchronization. ACM Trans. on Multimedia Comput. Commun. Appl. (TOMM), 10, $1 \mathrm{~s}(2014), 11$

[36] M. Obrist, A. N. Tuch, and K. Hornbaek. 2014. Opportunities for Odor: Experiences with Smell and Implications for Technology. In Proc. SIGCHI Conference on Human Factors in Computing Systems (CHI '14). ACM, New York, NY, USA, 2843-2852.

[37] K. Ooms, L. Dupont, L. Lapon, and S. Popelka. 2015. Accuracy and precision of fixation locations recorded with the low-cost Eye Tribe tracker in different experimental setups. Journal of eye movement research 8,1 .

[38] O. Palinko, A. L. Kun, A. Shyrokov, and P. Heeman. 2010. Estimating cognitive load using remote eye tracking in a driving simulator. In Proc. 2010 symposium on eye-tracking research \& applications. ACM, 141-144.

[39] E.B. Saleme, C.A.S. Santos, and G. Ghinea. 2019. A mulsemedia framework for delivering sensory effects to heterogeneous systems. Multimedia Systems, Springer, 25:421.

[40] H.S. Seo, E. Roidl, F. Müller, and S. Negoias. 2010. Odors enhance visual attention to congruent objects. Appetite, 54(3), 544-549.

[41] S. E. Stahl, H. S. An, D. M. Dinkel, J. M. Noble, and J. M. Lee. 2016. How accurate are the wrist-based heart rate monitors during walking and running activities? Are they accurate enough?. BMJ open sport \& exercise medicine, 2(1), e000106.

[42] Y. Sulema. 2016. Mulsemedia vs. Multimedia: State of the art and future trends. In Systems, Signals and Image Processing (IWSSIP), 2016 International Conference on . IEEE, 1-5.

[43] C. Timmerer, M. Waltl, B. Rainer, and N. Murray. 2014. Sensory Experience: Quality of Experience Beyond Audio-Visual. Springer International Publishing, Cham, 351-365.

[44] M.P. Wallen, S.R. Gomersall, S.E. Keating, U. Wisløff, and J.S. Coombes. 2016. Accuracy of Heart Rate Watches: Implications for Weight Management. Plos One. 11. pmid:27232714

[45] J. S. Winston, J. A. Gottfried, J. M. Kilner, and R. J. Dolan. 2005. Integrated neural representations of odor intensity and affective valence in human amygdala. Journal of Neuroscience 25, 39 (2005), 8903-8907.

[46] A. Yazdani, E. Kroupi, J.M. Vesin, and T. Ebrahimi. 2012. Electroencephalogram alterations during perception of pleasant and unpleasant odors. In Fourth International Workshop on Quality of Multimedia Experience, pp. 272-277.

[47] Z. Yuan, S. Chen, G. Ghinea, and G-M. Muntean. 2014. User quality of experience of mulsemedia applications. ACM Trans. on Multimedia Comput. Commun. Appl. (TOMM) 11, 1s (2014), 15.

[48] Z. Yuan, G. Ghinea, and G. Muntean. 2015. Beyond Multimedia Adaptation: Quality of Experience-Aware Multi-Sensorial Media Delivery. IEEE Transactions on Multimedia 17, 1, 104-117.

[49] Z. Yuan, G. Ghinea, and G-M. Muntean. 2014. Quality of experience study for multiple sensorial media delivery. In International Wireless Communications and Mobile Computing Conf. (IWCMC). 1142-1146.

[50] M. de Zambotti, , F. C. Baker, A.R. Willoughby, J.G. Godino, D. Wing, K. Patrick, and I.M. Colrain, 2016. Measures of sleep and cardiac functioning during sleep using a multi-sensory commercially-available wristband in adolescents. Physiology \& behavior, 158, 143-149.

[51] L. Zhang, S. Sun, B. Xing, J. Fu, and S. Yu. 2016. Exploring olfaction for enhancing multisensory and emotional game experience. In Int. Conf. on Tech. for E-Learning and Digital Entertainment. 111-121.

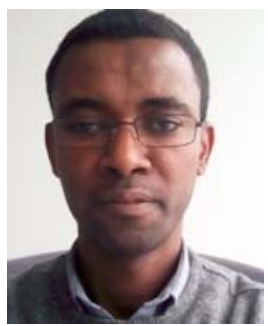

Gebremariam Mesfin is a 'Research assistant' in the Department of Computer Science at Brunel University London. He served as an 'Assistant professor' at Aksum University Ethiopia [2009-2016] and as a 'Lecturer' at Mekelle University Ethiopia [2004-2009]. He received a B.Sc. and M.Sc. in Computer Science, and Ph.D. in Information Technology (Software Engineering) from Addis Ababa University Ethiopia. Dr. Gebremariam has published research papers in software engineering, usability, crossplatform smartphone applications, multimedia, mulsemedia, Web service, service-oriented architecture, and e-learning.

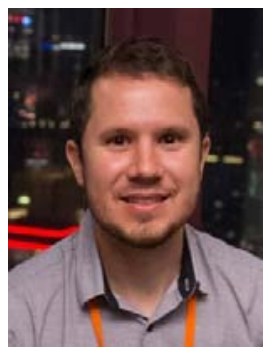

Estêvão Bissoli Saleme is a doctorate candidate in the Computer Science Department at Federal University of Espírito Santo, Brazil. He received the graduation degree in Information Systems from FAESA, Brazil, in 2008, the postgraduate degree in Software Engineering from the Federal University of Lavras, Brazil, in 2010, and the M.Sc. degree in Computer Science from the Federal University of Espírito Santo, Brazil, in 2015. His research interests include multimedia and human-computer interaction with a focus on digital multisensory systems (mainly mulsemedia), immersive technologies, and middleware and frameworks.

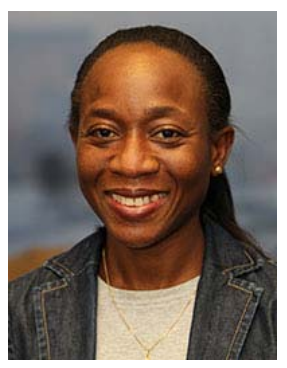

Oluwakemi A. Ademoye received a B.Sc. degree in Computer Science, in 1996, from the University of Benin, Nigeria; she then received a M.Sc. degree in Distributed Information Systems, and a Ph.D. degree in Information Systems \& Computing, from Brunel University, United Kingdom, in 2003 and 2008 respectively. She is currently a Lecturer in Computing, at the University of Wales Trinity Saint David. Her research interests focus on mulsemedia, distributed and mobile computing, and information visualization.

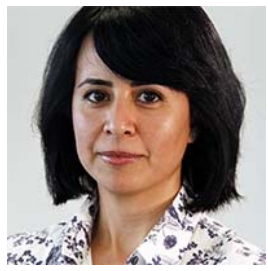

Elahe Kani-Zabihi is Lecturer in Creative Computing at the University of West London. Her research interests encompass the following areas: Digital Libraries; Human Computer Interaction; User-Centred Design; Mixed-Method research methodologies; Assistive Technology; Service Design and Creating innovative systems. 


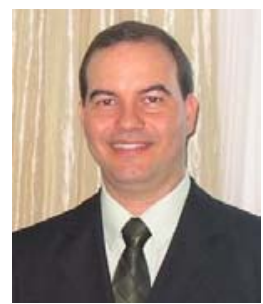

Celso A. S. Santos is a Professor in the Department of Informatics at Federal University of Espírito Santo, Brazil. He received the B.S. degree in Electrical Engineering from the Federal University of Espírito Santo in 1991, and the M.S. degree in Electrical Engineering (Electronic Systems) from the University of São Paulo, São Paulo-SP, Brazil, in 1994. In 1999, he received his Dr. degree from Université Paul Sabatier de Toulouse III, Toulouse, France. His recent research interests focus on multimedia systems and applications, synchronization, digital video, crowdsourcing systems.

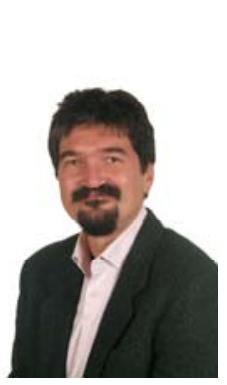

Gheorghiță Ghinea is a Professor of Mulsemedia Computing in the Computer Science Department at Brunel University, United Kingdom. He received the B.Sc. and B.Sc. (Hons) degrees in Computer Science and Mathematics, in 1993 and 1994, respectively, and the M.Sc. degree in Computer Science, in 1996, from the University of the Witwatersrand, Johannesburg, South Africa; he received the Ph.D. degree in Computer Science from the University of Reading, United Kingdom, in 2000. His work focuses on building adaptable cross-layer end-to-end communication systems incorporating user perceptual requirements. 